\title{
Measurement of empathy among Argentine cardiologists: Psychometrics and differences by age, gender, and subspecialty
}

\author{
Raúl Alfredo Borracci ${ }^{1}$, Hernán C. Doval ${ }^{2}$, Carmen Nuñez ${ }^{2}$, \\ Marisa Samarelli ${ }^{2}$, Susana Tamini ${ }^{2}$, Eduardo Tanus ${ }^{2}$ \\ ${ }^{1}$ Biostatistics, School of Medicine, Austral University, Buenos Aires, Argentina \\ ${ }^{2}$ Bioethics Committee of the Argentine Society of Cardiology, Buenos Aires, Argentina
}

\begin{abstract}
Background: Cardiologists are involved in the management of patients with multiple cardiovascular risk factors and chronic heart diseases, so empathy is a necessary feature to deal with them. The aim of the study was to evaluate the validity and reliability of the Spanish version of the Jefferson Scale of Physician Empathy (JSPE) among Argentine cardiologists and to explore the potential differences by age, gender, and subspecialty.

Methods: Between August and September 2012, we performed a survey in a non-randomized sample of 566 Spanish-speaking cardiologists of Argentina. A Principle Component Analysis (PCA) was used to explore the link between observed variables and latent variables in order to identify the factor structure. The PCA criteria for identifying the factor structure were examined with the Kaiser-Meyer-Olkin (KMO) analysis.

Results: The KMO measure of sampling adequacy was 0.86 and Bartlett's test of sphericity was highly significant $(p=0.000)$, determining the suitability of the data set for factor analysis. The PCA of 20 items yielded a three factor model that accounted for $40.6 \%$ of the variance. The JSPE mean rank score for women was 307.9 vs. 275.0 for men $(p=0.017)$. The comparison of mean rank score according to age (quartiles) showed a significant relation between older age and empathy. No difference was found when the mean rank scores were compared by respondent subspecialty.

Conclusions: JSPE provides a valid and reliable scale to measure Argentine cardiologists' attitudes towards empathy. Female cardiologists seem to be more empathic than their male colleagues, and a positive relationship between age and empathy was found. (Cardiol J 2015; 22, 1: 52-56)
\end{abstract}

Key words: empathy, Jefferson Scale of Physician Empathy, cardiologists, Argentine

Address for correspondence: Dr Raúl A. Borracci, Biostatistics, School of Medicine, Austral University, La Pampa 3030, 1428 Buenos Aires, Argentina, tel: (54-011) 4784-5917, fax: (54-11) 4961-6027, e-mail: raborracci@gmail.com Received: 07.02.2014 Accepted: 02.06.2014 


\section{Introduction}

Most patients agree that being empathic is a valuable trait in their physicians, and recent research suggests that emotional communication between patient and physician positively influences healing [1-4]. For instance, physician empathy seems to be associated with better clinical outcomes for patients with diabetes $[5,6]$.

The quantitative measurement of empathy in health care professionals is not easy, but it is considered crucial to evaluate the effectiveness of educational interventions aimed at promoting empathy $[7,8]$. The Jefferson Scale of Physician Empathy (JSPE) is the most widely used tool to measure empathy in the context of patient care $[9,10]$. Nevertheless, its psychometric properties such as validity and reliability must be proved and culturally adapted for the specific population of interest. The JSPE has been translated into 42 languages and recently these different versions were used for assessing empathy among American [9], English [8], Italian [11], Japanese [12], Mexican [13], French [14], German [15], Polish [16], Korean [17] and Iranian [18] physicians or medical students.

Empathic engagement is particularly important in specialties which treat chronically ill patients requiring continuity of care. These patients need a solid physician-patient relationship to achieve an adequate adherence to medication. Cardiologists are involved in the management of patients with multiple cardiovascular risk factors and chronic heart diseases, so empathy is a necessary feature to deal with them.

The purpose of this study was to evaluate the validity and reliability of the Spanish version of the JSPE among Argentine cardiologists and to explore the potential differences by age, gender, and subspecialty.

\section{Methods}

Between August and September 2012, we conducted a survey in a non-randomized sample of 566 Spanish-speaking cardiologists included in the Argentine Society of Cardiology register, proportionally representing the Argentine distribution of cardiology specialists. They answered via e-mail a Spanish version of the JSPE questionnaire previously validated by Alcorta-Garza et al. [13]. The participation in the study was anonymous and voluntary, and the questionnaire took less than $10 \mathrm{~min}$ to be completed. The JSPE is a self-administrated 20-item scale designed to measure physician empathy in the context of patient care and doctor-patient relationship [9, 10]. The level of empathy for each item is calculated on a 7-point Likert scale from 1 (strongly disagree) to 7 (strongly agree). Additionally, information about age, gender, and subspecialty provided by respondents was required to complete the form.

The study was reviewed and approved by the local bioethical committee of the Argentine Society of Cardiology.

\section{Statistical analysis}

First, to facilitate interpretation and analysis, responses for negative items were reversed. Due to the question structure, the occurrence of ceiling effect was expected, and the non-Gaussian distribution of empathy scores was assessed with the Kolmogorov-Smirnov goodness-of-fit test. The median score was adopted as representative value; nevertheless, mean and standard deviation (SD) were included in the factor analysis. Although the underlying JSPE components were studied in other populations, a Principle Component Analysis (PCA) was locally used to explore the link between observed variables (items) and latent variables (factors) in order to identify factor structure. Only factors with eigenvalues greater than 1.25 were retained, and factor coefficients greater than 0.40 were required for the interpretation of factor structure, using Varimax rotation. The PCA criteria for identifying factor structure were examined with the Kaiser-Meyer-Olkin (KMO) analysis. To establish whether the data set was suitable for the factor analysis, a KMO index greater than 0.50 was adopted [19, 20]. Previous research determined that the JSPE model represents empathy as a multidimensional construct with perspective taking, compassionate care, and emotional detachment as conceptually independent factors [7, 12]. Finally, the internal consistency of the scale was assessed with Cronbach's alpha, and a value greater than 0.70 was considered to be an acceptable reliability coefficient. Two-tailed Mann-Whitney U and Kruskal-Wallis non-parametric tests were used to compare non-normal score distributions. All statistical analyses were performed using SPSS 17.0 software.

\section{Results}

Five hundred and sixty six of 900 surveyed cardiologists $(62.9 \%)$ completed the questionnaire. Mean age was 51.4 (SD 11.4, range 24-84), 75.5\% were male, $80.9 \%(\mathrm{n}=458$ ) were clinical cardiologists and the rest belonged to other subspecialties (imaging, electrophysiology, critical care, interventional cardiology or cardiac surgery). 
Table 1. Principle component analysis of items in the Jefferson Scale of Physician Empathy $(n=566)$.

\begin{tabular}{|c|c|c|c|c|c|c|}
\hline No. item & Factor 1 & Factor 2 & Factor 3 & $h^{2}$ & Mean & SD \\
\hline 2 & 0.59 & - & - & 0.35 & 6.52 & 1.18 \\
\hline 4 & 0.64 & - & - & 0.43 & 6.25 & 1.37 \\
\hline 10 & 0.67 & - & - & 0.49 & 6.10 & 1.29 \\
\hline 13 & 0.66 & - & - & 0.48 & 6.05 & 1.32 \\
\hline 15 & 0.58 & - & - & 0.42 & 6.15 & 1.40 \\
\hline 16 & 0.59 & - & - & 0.51 & 6.30 & 1.14 \\
\hline 20 & 0.59 & - & - & 0.45 & 6.52 & 1.09 \\
\hline $1 *$ & - & 0.49 & - & 0.34 & 5.78 & 2.09 \\
\hline $7^{*}$ & - & 0.52 & - & 0.30 & 5.59 & 1.99 \\
\hline $8^{*}$ & - & 0.53 & - & 0.39 & 6.08 & 1.58 \\
\hline $11^{*}$ & - & 0.55 & - & 0.42 & 6.28 & 1.31 \\
\hline $12^{*}$ & - & 0.57 & - & 0.33 & 6.06 & 1.81 \\
\hline $14^{*}$ & - & 0.58 & - & 0.36 & 6.52 & 1.25 \\
\hline $19 *$ & - & 0.47 & - & 0.32 & 6.44 & 1.28 \\
\hline $3^{*}$ & - & - & 0.70 & 0.53 & 5.29 & 1.76 \\
\hline $6^{*}$ & - & - & 0.54 & 0.43 & 5.79 & 1.63 \\
\hline 9 & - & - & 0.53 & 0.55 & 5.57 & 1.68 \\
\hline 17 & - & - & 0.55 & 0.52 & 5.38 & 1.69 \\
\hline $18^{*}$ & - & - & 0.41 & 0.18 & 3.83 & 2.04 \\
\hline Eigenvalues & 4.89 & 1.92 & 1.31 & & & \\
\hline$\%$ of variance & 24.40 & 9.59 & 6.56 & & & \\
\hline Alpha & 0.792 & 0.620 & 0.613 & & & \\
\hline
\end{tabular}

*Items were reverse scored (strongly agree $=1$, strongly disagree $=7$ ); Factor 1 - perspective taking; Factor 2 - compassionate care; Factor 3 - emotional detachment; Factor pattern coefficients $\leq 0.40$ were omitted; $\mathrm{h}^{2}-$ communalities of each item

The distribution of JSPE scores was J-shaped and $59.5 \%(n=337)$ of physicians obtained the maximum score. In order to counteract the severely skewed distribution and the extreme ceiling effect, a non-parametric analysis was adopted.

The KMO measure of sampling adequacy was 0.86 and Bartlett's test of sphericity was highly significant $\left(\chi_{(190)}^{2}=2476 ; p=0.000\right)$, determining the suitability of the data set for the factor analysis. The PCA of 20 items yielded a 3 -factor model that accounted for $40.6 \%$ of the variance (Table 1 ). The first factor denoted by 'perspective taking' was explained by 8 items; the second factor identified as 'compassionate care' was described by 7 items; and the third factor labeled 'emotional detachment or standing in the patient's shoes' contained 5 items. These 3 dimensions were used to compare the results by gender, age, and subspecialty.

The JSPE mean rank score for women was 307.9 vs. 275.0 for men $(p=0.017)$. The same comparison by gender and divided into the 3 dimensions is shown in Table 2. The comparison of mean rank score according to age (quartiles) showed a significant relation between older age and empathy (Table 3). On the contrary, no difference
Table 2. Comparison between male $(n=419)$ and female $(n=147)$ responses on the Jefferson Scale of Physician Empathy (mean rank).

\begin{tabular}{lccc}
\hline Dimension & Male & Female & $\mathrm{P}^{*}$ \\
\hline $\begin{array}{l}\text { Perspective taking } \\
\text { Compassionate }\end{array}$ & 278.3 & 298.3 & 0.149 \\
$\begin{array}{l}\text { care } \\
\begin{array}{l}\text { Emotional } \\
\text { detachment }\end{array}\end{array}$ & 286.2 & 275.9 & 0.501 \\
Global ranking & 275.0 & 307.9 & 0.017 \\
\hline
\end{tabular}

*Two-tailed Mann-Whitney U test

was found when mean rank score was compared by respondent subspecialty (clinical cardiology $(\mathrm{n}=458)$ vs. the rest $(\mathrm{n}=108, \mathrm{p}=0.626)$.

\section{Discussion}

The results of this study support the JSPE reliability and validity for measuring empathy among Argentine cardiologists. The resulting factor structure of the Spanish version responded by our physicians was very similar to that reported 
Table 3. Comparison of responses on the Jefferson Scale of Physician Empathy according to age (mean rank).

\begin{tabular}{lccccc}
\hline Dimension & $<$ 43 years & 43-53 years & 53-59 years & $>$ 59 years & P* \\
\hline Perspective taking & 230.9 & 273.9 & 304.1 & 321.8 & 0.000 \\
Compassionate care & 261.5 & 286.1 & 303.4 & 315.1 & 0.005 \\
Emotional detachment & 251.8 & 275.8 & 285.5 & 320.3 & 0.005 \\
Global ranking & 238.7 & 280.2 & 294.5 & 329.3 & 0.000 \\
\hline
\end{tabular}

*Kruskal-Wallis test

for Mexican medical students [13], and somewhat different to that communicated for Anglo-Saxons $[8,9]$. The multidimensional concept involving empathy and cross-cultural contrasts would explain these differences. The PCA did not exclude any item and identified the 3-factor model previously described. Likewise, psychometric data like KMO index, sphericity, variance, and eigenvalues assigned to each factor were similar to those reported in other communications $[8,13]$.

In opposition to other authors, our statistical approach included a non-Gaussian treatment of data and a non-parametric testing in order to counteract the ceiling effect of skewed distributions. These methods allowed us a better approximation to match for gender and age differences.

The prevailing view seems to be that female physicians have higher JSPE empathy scores compared to their male colleagues. In our study, female cardiologists had a higher global ranking than males, particularly based on the 'perspective taking' and the 'compassionate care' factors. Similar results were reported by Tavakol et al. [8] in United Kingdom medical students, where the empathy scores of female students were significantly different on the 3 dimensions. The observed gender differences may have implications for sustaining empathy, especially when taking into account that the number of female physicians in Argentina has increased rapidly in the past years [21].

We did not find differences between empathy scores in relation to cardiology subspecialties. Nevertheless, other authors have demonstrated that physicians who practice in "people-oriented" specialties like psychiatry, pediatrics, internal and family medicine obtain a significantly higher mean empathy score than their counterparts in "procedure or technology-oriented" specialties, as surgery, radiology or obstetrics $[10,12]$.

Previous studies could not demonstrate a correlation between age and empathy. It is possible that the true relationship between these variables cannot be fully captured with the extreme ceiling effect of empathy scores, unless a non-parametric approach is used. When we adopted the median score distributed by age quartiles to counterbalance the severely skewed data distribution, a significant relationship between older age and empathy was found in the 3 dimensions. With control for gender, older physicians seem to be more empathic than younger doctors. A possible explanation would be that empathy could improve with increasing practice and clinical experience. On the other hand, younger physicians could despise the value of empathy in favor of a better technology-based medical practice. Although Barnsley et al. [22] found that recent graduates were significantly more likely to report an empathetic communication style with patients, to the best of our knowledge no other author has reported a close relationship between empathy and physician age. These findings demonstrate that it is necessary to improve the communication skills on empathy among young physicians, in both undergraduate and graduate educational programs. Teaching younger cardiologists empathy is essential to understand the patient's perspective and develop a better medical professionalism.

\section{Limitations of the study}

The principal limitation of this study was the reduced response rate of the questionnaire and the non-probabilistic characteristic of the sample, which prevented extending the results to all Argentine cardiologists. Since more than $20 \%$ of selected cardiologists did not participate in the survey, the missing data could have an important effect on the generalization of the collected results. Argentine Society of Cardiology register contains 2,887 cardiologists and the total number of specialists all over the country probably would be double as many; that is why the sample size could constitute a weak point in our data. Moreover, the self-reported data of the JSPE survey may limit the validity of the findings, since respondents may 
over or underestimate their own empathic practice. Although the JSPE is a frequently used method to assess "empathy", it has some important limitations. Perhaps the most important is that JSPE allows an evaluation of the empathy perceived from the physicians' viewpoint and on the contrary, it does not allow any evaluation of the perception of empathy from the patients, who are the other half of the action. This point must be stressed, since patients have been shown to interpret empathy in a different fashion as physicians do [23].

\section{Conclusions}

In conclusion, this study showed that the JSPE provides a valid and reliable scale to measure Argentine cardiologists' attitudes towards empathy. Female cardiologists seem to be more empathic than their male colleagues, at least in 2 of the 3 factors of the multidimensional empathy structure. Finally, a positive relationship between age and empathy was found, and older cardiologists ranked better in the scale than younger doctors. Since empathy has been linked not only to patient satisfaction, but also to clinical competence, this unexpected finding must be considered to promote empathic skills in young physicians, in both undergraduate and graduate medical education.

Conflict of interest: All authors are related to the Bioethic Committee of the Argentine Society of Cardiology, and this fact could be considered a potential conflict of interest regarding this study.

\section{References}

1. Halpern J. What is clinical empathy? J Gen Intern Med, 2003; 18: 670-674.

2. Kim SS, Kaplowitz S, Johnston MV. The effects of physician empathy on patient satisfaction and compliance. Eval Health Prof, 2004; 27: 237-251.

3. Street RI Jr, Makoul G, Arora NK, Epstein RM. How does communication heal? Pathways linking clinician-patient communication to health outcomes. Patient Educ Couns, 2009; 74: 295-301.

4. Rakel DP, Hoeft TJ, Barrett BP, Chewning BA, Craig BM, Niu M. Practitioner empathy and the duration of the common cold. Fam Med, 2009; 41: 494-501.

5. Hojat M, Louis DZ, Markham FW, Wender R, Rabinowitz C, Gonnella JS. Physicians' empathy and clinical outcomes for diabetic patients. Acad Med, 2011; 86: 359-364.

6. Del Canale S, Louis DZ, Maio V, Wang X, Rossi G, Hojat M, Gonnella JS. The relationship between physician empathy and disease complications: An empirical study of primary care physicians and their diabetic patients in Parma, Italy. Acad Med, 2012; 87: 1243-1249.
7. Hojat M. Ten approaches for enhancing empathy in health and human services cultures. J Health Hum Serv Adm, 2009; 31: 412-450.

8. Tavakol S, Dennick R, Tavakol M. Psychometric properties and confirmatory factor analysis of the Jefferson Scale of Physician Empathy. BMC Medical Education, 2011; 1: 54-61.

9. Hojat M, Mangione S, Nasca TJ et al. The Jefferson Scale of Physician Empathy: Development and preliminary psychometric data. Educ Psychol Meas, 2001; 61: 349-365.

10. Hojat M, Gonnella JS, Nasca TJ, Mangione S, Vergare M, Magee M. Physician empathy: Definition, components, measurement, and relationship to gender and specialty. Am J Psychiatry, 2002; 159: 1563-1569.

11. Di Lillo M, Cicchetti A, Lo Scalzo A, Taroni F, Hojat M. The Jefferson Scale of Physicians Empathy: Preliminary psychometrics and group comparisons in Italian physicians. Acad Med, 2009; 84: 1198-1202.

12. Kataoka HU, Koide N, Hojat M, Gonnella JS. Measurement and correlates of empathy among female Japanese physicians. BMC Med Educ, 2012; 12: 48.

13. Alcorta-Garza A, Gonzalez-Guerrero JF, Tavitas-Herrera SE, Rodriguez-Lara FJ, Hojat M. Validation of the Jefferson Scale of Physician Empathy in Mexican medical students [in Spanish]. Salud Mental (Mex), 2005; 28: 57-62.

14. Lelorain S, Sultan S, Zenasni F, Catu-Pinault A, Jaury P, Boujut E, Rigal L. Empathic concern and professional characteristics associated with clinical empathy in French general practitioners. Eur J Gen Pract, 2013; 19: 23-28.

15. Neumann M, Scheffer C, Tauschel D, Lutz G, Wirtz M, Edelhäuser F. Physician empathy: Definition, outcome-relevance and its measurement in patient care and medical education. GMS Z Med Ausbild, 2012; 29 (1): Doc11. DOI: 10.3205/zma000781. Epub 2012 Feb 15.

16. Kliszcz J, Nowicka-Sauer K, Trzeciak B, Nowak P, Sadowska A. Empathy in health care providers: Validation study of the Polish version of the Jefferson Scale of Empathy. Adv Med Sci, 2006; 51: 219-225.

17. Suh DH, Hong JS, Lee DH, Gonnella JS, Hojat M. The Jefferson Scale of Physician Empathy: A preliminary psychometric study and group comparisons in Korean physicians. Med Teach 2012; 34: e464-e468.

18. Shariat SV, Eshtad E, Ansari S. Empathy and its correlates in Iranian physicians: A preliminary psychometric study of the Jefferson Scale of Physician Empathy. Med Teach, 2010; 32: e417-e421.

19. Henson R, Roberts J. Use of exploratory factor analysis in published research: Common errors and some comment on improved practice. Educ Psychol Meas, 2006; 66: 393-416.

20. Hogarty K, Hines C, Kromrey J, Ferron J, Mumford K. The quality of factor solutions in exploratory factor analysis: The influence of sample size, communality, and overdetermination. Educ Psychol Meas, 2005; 65: 202-226.

21. Borracci RA, Pittaluga RD, Manente D, Giorgi MA, Rubio M. Medical students' attitudes about professional practice. Medicina (Buenos Aires), 2009; 69: 607-611.

22. Barnsley J, Williams AP, Cockerill R, Tanner J. Physician characteristics and the physician-patient relationship Impact of sex, year of graduation, and specialty. Can Fam Physician, 1999; 45: 935-942.

23. Kane GC, Gotto JL, Mangione S, West S, Hojat M. Jefferson Scale of Patient's Perceptions of Physician Empathy: Preliminary Psychometric Data. Croat Med J, 2007; 48: 81-86. 